\title{
Local response to light excitation in the charge-ordered phase of $(\mathrm{EDO}-\mathrm{TTF})_{2} \mathrm{SbF}_{6}$
}

\author{
Marina Servol, ${ }^{1, *}$ Nicolas Moisan, ${ }^{1}$ Eric Collet, ${ }^{1}$ Hervé Cailleau, ${ }^{1}$ Wawrzyniec Kaszub,${ }^{1}$ Loïc Toupet, ${ }^{1}$ Davide Boschetto, ${ }^{2}$ \\ Tadahiko Ishikawa, ${ }^{3}$ Alain Moréac, ${ }^{1}$ Shinya Koshihara, ${ }^{3}$ Mitsuhiko Maesato, ${ }^{4}$ Mikio Uruichi, ${ }^{5}$ Xiangfeng Shao,,${ }^{6, \dagger}$ \\ Yoshiaki Nakano, ${ }^{6}$ Hideki Yamochi, ${ }^{6}$ Gunzi Saito,,${ }^{4,6}+$ and Maciej Lorenc ${ }^{1}$ \\ ${ }^{1}$ Institut de Physique de Rennes, UMR 6251 CNRS-University Rennes 1 campus Beaulieu, Rennes, 35042, France \\ ${ }^{2}$ Laboratoire d'Optique Appliquée, ENSTA ParisTech, CNRS, Ecole polytechnique, Université Paris-Saclay, 828 boulevard des Maréchaux, \\ 91762 Palaiseau cedex, France \\ ${ }^{3}$ Department of Materials Science, Tokyo Inst. of Technology, 2-12-1, Oh-okayama, Meguro, Tokyo, 152-8551, Japan \\ ${ }^{4}$ Division of Chemistry, Graduate School of Science, Kyoto University, Sakyo-ku, Kyoto, 606-8501, Japan \\ ${ }^{5}$ Institute for Molecular Science, Myodaiji, Okazaki 444-8585, Japan \\ ${ }^{6}$ Research Center for Low Temperature and Materials Sciences, Kyoto University, Sakyo-ku, Kyoto, 606-8502, Japan
}

(Received 15 October 2014; published 24 July 2015)

\begin{abstract}
The family of materials (EDO-TTF $)_{2} \mathrm{XF}_{6}$ represents quasi-one-dimensional quarter filled systems exhibiting insulator-to-metal (I-M) phase transition at thermal equilibrium. (EDO-TTF) ${ }_{2} \mathrm{PF}_{6}$ is known to undergo a photoinduced I-M conversion with cooperative response to light excitation. Here we use femtosecond pump-probe experiments to study the photoresponse of (EDO-TTF $)_{2} \mathrm{SbF}_{6}$ made of a larger counteranion $\mathrm{SbF}_{6}$ compared to the well studied (EDO-TTF $)_{2} \mathrm{PF}_{6}$. In the early stage of the photoinduced process, we reveal a multicomponent coherent oscillating feature. The evolution of this feature with excitation density and temperature points to the local nature of the photoswitching in $(\mathrm{EDO}-\mathrm{TTF})_{2} \mathrm{SbF}_{6}$. At longer time scale, we did not detect the features associated with the transformation to the $\mathrm{M}$ phase, albeit observed in the $\mathrm{PF}_{6}$ derivative. We propose a scenario whereby the bigger size of the counteranion in $(\mathrm{EDO}-\mathrm{TTF})_{2} \mathrm{SbF}_{6}$ hinders the establishment of this transformation at macroscopic scale.
\end{abstract}

DOI: 10.1103/PhysRevB.92.024304

PACS number(s): 78.47.-p, 82.53.Xa, 31.70.Ks, 42.70.Gi

\section{INTRODUCTION}

The development of ultrafast time-resolved spectroscopies, and especially the pump-probe techniques, gives new possibilities for tracking and controlling dynamical transformations in the solid state on ultrashort time scale. An intense pump laser pulse prepares the system in a highly excited state involving a bunch of excited electrons, and a probe pulse monitors their temporal evolution thereby providing information on the triggered collective and/or cooperative phenomena. This technique makes it possible to track coherent atomic/molecular motions and physical transformations of a material in real time. During the last decade, the field of ultrafast photoinduced transformation has been expanding rapidly along diverse directions: correlated electrons, charge density waves, magnetism, molecular switching, etc. [1-4]. A typical example is provided by a quasi-one-dimensional quarter filled organic conductor (EDO-TTF $)_{2} \mathrm{PF}_{6}$ where EDO-TTF is the abbreviation of the electron donor (D) molecule ethylenedioxytetrathiafulvalene. It exhibits first-order insulator-to-metal (I-M) phase transition at thermal equilibrium and a gigantic ultrafast change of reflectivity under irradiation by a laser pulse in the I phase [5].

\footnotetext{
*To whom correspondence should be addressed: marina.servol@univ-rennes1.fr

${ }^{\dagger}$ Present address: State Key Laboratory of Applied Organic Chemistry, Lanzhou University, Tianshui Southern Road 222, Lanzhou 730000, Gansu Province, People's Republic of China.

${ }^{\ddagger}$ Present address: Faculty of Agriculture, Meijo University, Tempaku-ku, Nagoya 468-8502, Japan and Toyota Physical and Chemical Research Institute, 41-1, Yokomichi, Nagakute, Aichi 480-1192, Japan.
}

It was shown by time-resolved optical [6,7] and vibrational spectroscopy [8] as well as ultrafast electron diffraction [9] that the photoinduced transformation proceeds stepwise. The initial electronic excited state is trapped at the molecular level within 40 fs following the electron-phonon coupled precursor state [7], while the crystal transformation is driven by slower propagation at the $100 \mathrm{ps}$ time scale. Actually, different isostructural compounds of the same (EDO-TTF) ${ }_{2} \mathrm{XF}_{6}$ family, where $\mathrm{X}$ stands for $\mathrm{P}$, As, or $\mathrm{Sb}$, have already been synthesized. They undergo similar I-M phase transition at thermal equilibrium. However, the transition temperature is decreased and the width of thermal hysteresis is increased with increasing anion size [10].

The phase transition at thermal equilibrium of (EDO-TTF $)_{2} \mathrm{PF}_{6}$ between $\mathrm{M}$ and I phases reveals different facets such as Peierls distortion and charge ordering, as well as a significant reduction of the orientational disorder of $\mathrm{XF}_{6}$ counteranions. The main feature is the charge order along donor stack $\left(\mathrm{D}^{0} \mathrm{D}^{1} \mathrm{D}^{1} \mathrm{D}^{0}\right)$, in contrast to the more common order $\left(\mathrm{D}^{1} \mathrm{D}^{0} \mathrm{D}^{1} \mathrm{D}^{0}\right)$ [11-13]. For photoinduced transformation, the similarity between relaxation dynamics of reflectivity and optical conductivity, both occurring on the microsecond scale, indicates that a transient metallic state is generated on this time scale [5]. Additional experimental measurements [6] showed that the reflectivity spectrum of the photoinduced state differs on the ultrashort time scale from that of the metallic state at thermal equilibrium, when seen over a broad energy range from $69 \mathrm{meV}$ to $2.1 \mathrm{eV}$. These results, combined with theoretical investigations, demonstrated that a photoexcitation of the charge-ordered insulator $\left(\mathrm{D}^{0} \mathrm{D}^{1} \mathrm{D}^{1} \mathrm{D}^{0}\right)$ into its charge-transfer band around $1.58 \mathrm{eV}$ does not create directly a metallic phase through charge melting $\left(\mathrm{D}^{0.5} \mathrm{D}^{0.5} \mathrm{D}^{0.5} \mathrm{D}^{0.5}\right)$ but rather leads to a photoinduced $\left(\mathrm{D}^{1} \mathrm{D}^{0} \mathrm{D}^{1} \mathrm{D}^{0}\right)$ state at the picosecond time 
scale. Recently, time resolved vibrational spectroscopy [8] and ultrafast electron diffraction measurements [9] unveiled that the photoinduced phase similar to the metallic one of the high temperature phase is only reached around $100 \mathrm{ps}$. During the relaxation from the photoexcited electronic state to the photoinduced $\left(\mathrm{D}^{1} \mathrm{D}^{0} \mathrm{D}^{1} \mathrm{D}^{0}\right)$ state, a coherent phonon with frequency around $2 \mathrm{THz}$ was also observed [5]. In parallel to these experimental studies [14], theoretical attempts have been undertaken to explain the unusual $\left(\mathrm{D}^{0} \mathrm{D}^{1} \mathrm{D}^{1} \mathrm{D}^{0}\right)$ order of the insulating phase and the photoinduced phase transition [6,13-17].

The question whether the anion size favors either local or three-dimensional (3D) photoinduced transformation has hitherto not been addressed. In this paper we investigate the response to femtosecond laser excitation of the (EDO-TTF $)_{2} \mathrm{SbF}_{6}$ derivative, which is isostructural to (EDO-TTF $)_{2} \mathrm{PF}_{6}$ but for a larger anion size. In particular, the coherent dynamics at the picosecond time scale is studied, revealing the generation of several coherent phonons. In Sec. II the experimental procedure and especially the femtosecond pump-probe experiment is presented. In Sec. III we describe the insulating to metal phase transition occurring at thermal equilibrium in the $\mathrm{SbF}_{6}$ derivative by using structural and electronic probes for tracking the degrees of freedom involved. In parallel, we highlight the differences with the case of (EDO-TTF) ${ }_{2} \mathrm{PF}_{6}$. In Sec. IV the ultrafast dynamics is presented, and in Sec. V we provide a comprehensive analysis of the optical data obtained together with a phenomenological description of the anion size effect.

\section{EXPERIMENTAL DETAILS}

Different complementary experiments were used for characterizing the I-M phase transition of (EDO-TTF $)_{2} \mathrm{SbF}_{6}$ at thermal equilibrium. X-ray diffraction was performed on single crystals with typical sizes of $600 \times 200 \times 100 \mu \mathrm{m}^{3}$. The diffraction experiments were carried out on a Xcalibur 3 four-circles diffractometer (Oxford Diffraction) equipped with a two-dimensional (2D) sapphire 3 CCD detector with Mo $K \alpha$ radiation $(\lambda=0.71069 \AA)$. The single crystals were placed in Oxford Cryosystem nitrogen-flow cryostat with a temperature stability of $0.1 \mathrm{~K}$. Crystals were initially flash-cooled to $100 \mathrm{~K}$ to avoid sample damage in the vicinity of the first-order phase transition and then heated to a set temperature. Although the flash-cooling procedure could create phase domains, none was observed. The unit cell parameters and the data reduction were obtained with CrysAlis software from Oxford Diffraction [18]. The structures (Fig. 1) were solved with SIR-97 [19] and refined with SHELXL97 [20]. Magnetic susceptibility of the polycrystalline sample was measured with a Quantum Design MPMS-XL system and corrected for both the magnetization of the sample holder and the diamagnetic contribution of the sample itself. The estimation of the diamagnetic contribution was done using Pascal's constants. Raman spectra measurements were performed with an XY DILOR triple monochromator. Optical reflectivity data were obtained with a tungsten-halogen lamp coupled to a monochromator (Spectral Products) for the light source, and a photodiode with a lock-in amplifier (Signal recovery) for detection. (a)

$250 \mathrm{~K}$

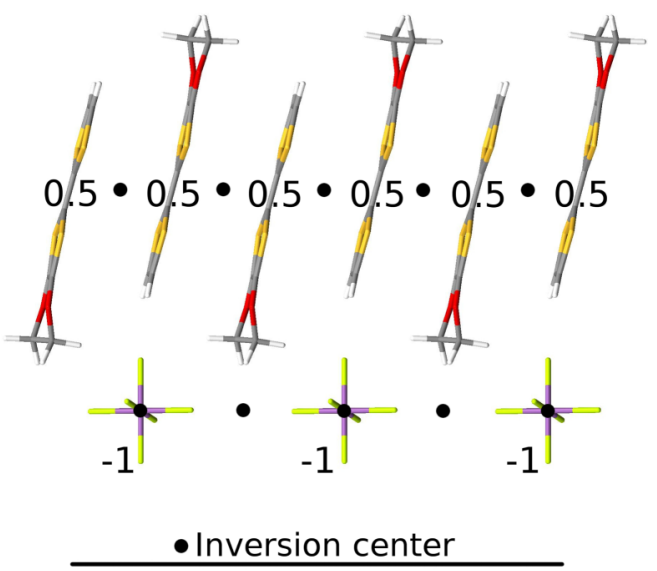

(b)

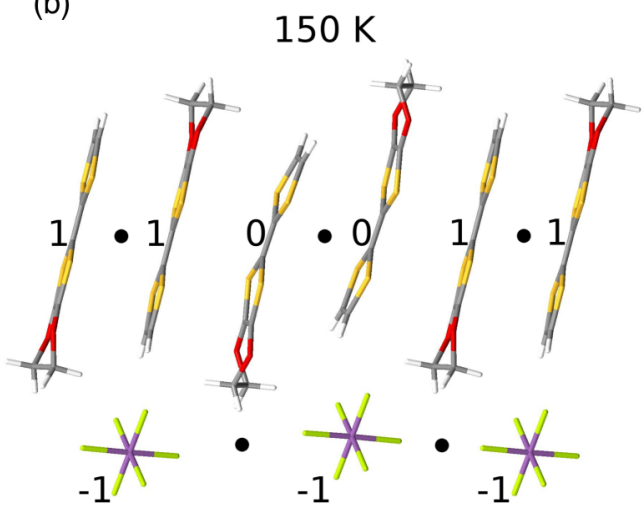

FIG. 1. (Color online) Crystal structures of the M (a) and I phase (b) and the corresponding schematic charge distribution on the $\mathrm{D}$ molecules. In the I phase, the symmetry breaking resulting from charge ordering involves important structural reorganization with, for example, the bending of the neutral molecules. Due to the cell doubling along the stack, the inversion symmetry is lowered, and the charge ordering accompanied by important modifications of the orientation of the $\mathrm{SbF}_{6}^{-}$anions is observed.

In order to investigate the dynamics of the photoinduced response of (EDO-TTF $)_{2} \mathrm{SbF}_{6}$, optical pump-probe experiments were performed. The laser system used consisted of a Ti:sapphire oscillator (MIRA, Coherent) delivering a $76 \mathrm{MHz}$ train of $40 \mathrm{fs}$ pulses centered at $1.55 \mathrm{eV}$. This train is amplified at $1 \mathrm{kHz}$ by a regenerative amplifier (LEGEND, Coherent) to $2.2 \mathrm{~mJ}$. The beam is split into two, the pump and the probe beam, each pumping an optical parametric amplifier (TOPAS, Light Conversion) [21]. The wavelength of the two beams can be tuned from 0.41 to $6.2 \mathrm{eV}$. The pulse duration after the TOPAS is estimated to be $50 \mathrm{fs}$. The pump energy was set from 4 to $1400 \mu \mathrm{J} \mathrm{cm}^{-2}$ ( 1 photon per 18000 to 55 molecules [5]) due to the sample damage above $1500 \mu \mathrm{J} \mathrm{cm}^{-2}$. The probe fluency used was set to $1 \mu \mathrm{J} \mathrm{cm}^{-2}$, so that it did not affect the sample response.

The pump-probe delay was set by a linear stage (IMS, Newport). In order to increase the temporal resolution, a collinear geometry between pump and probe was chosen: the recombination of the two beams was done with a wedged half mirror. The cross correlation between pump and probe gave 
final temporal resolution with full width at half maximum (FWHM) of around $80 \mathrm{fs}$.

The pump and the probe were focused on the crystal by lenses of 500 and $200 \mathrm{~mm}$ focal length, respectively, which resulted in beam waists of 380 and $150 \mu \mathrm{m}$ at FWHM. A larger spot was chosen for the pump, so that any small displacement of the pump beam would be negligible for the excitation of the probed part of the crystal. The polarization of both, pump and probe, were parallel to the EDO-TTF stacking axis of the crystal. An Oxford Cryosystem nitrogen-flow cryostat was used to control the temperature, and the same flash-cooling procedure was applied as described above.

Finally, we detected the relative change in reflectivity with a phase sensitive lock-in detection (Stanford research). To perform this detection, the pumping rate of the crystal was halved to $500 \mathrm{~Hz}$ by an optical chopper. The $1 \mathrm{kHz}$ probe impinged on the crystal excited at $500 \mathrm{~Hz}$ and the photodiode measured a reflectivity signal modulated at this latter frequency. The role of the lock-in amplifier was to recover the $500 \mathrm{~Hz}$ modulation signal. In order to take shotto-shot fluctuations of the laser into account, the reflectivity signal was balanced with a reference signal sampled before the sample. At best, this detection method allowed sensitivity as high as $10^{-5}$.

\section{I-M PHASE TRANSITION AT THERMAL EQUILIBRIUM}

The I-M transition in (EDO-TTF $)_{2} \mathrm{SbF}_{6}$ [22] at thermal equilibrium is similar to that observed for (EDO-TTF $)_{2} \mathrm{PF}_{6}$. In the high temperature $\mathrm{M}$ phase (Fig. 1), the space group is $P \overline{1}$ and in the dimers formed by two head-to-tail D molecules, both $\mathrm{D}$ are equivalent by inversion symmetry located in-between them. As a result, the electron kept by one counteranion corresponds to one hole distributed over two D, with formal charges +0.5 equal by symmetry. In the insulating phase, a symmetry breaking takes place with a cell doubling and the loss of half of the inversion centers. This generates two independent pairs of donor dimers centered on inversion symmetry with two different formal charges. These charges are empirically estimated from intermolecular bond length obtained by x-ray diffraction $[11,23,24]$ or by maximum entropy method [12]. The alternation of bent neutral and flat ionized dimers is characteristic of this charge order as observed at $150 \mathrm{~K}$ in Fig. 1. In other words, the symmetry breaking is associated with charge ordering process along the $\mathrm{D}$ stack. It can be schematically represented by $\left(\mathrm{D}^{0.5} \mathrm{D}^{0.5} \mathrm{D}^{0.5} \mathrm{D}^{0.5}\right)$ in the $\mathrm{M}$ phase, and by $\left(\mathrm{D}^{0} \mathrm{D}^{1} \mathrm{D}^{1} \mathrm{D}^{0}\right)$ in the I phase.

However, some structural differences appear in the intermolecular organization between the $\mathrm{PF}_{6}$ and $\mathrm{SbF}_{6}$ derivatives [6]. The main feature is that the larger size of the $\mathrm{SbF}_{6}{ }^{-}$ anions results in a bigger unit cell. The distance $b$ between $\mathrm{D}$ molecules is slightly longer for the $\mathrm{SbF}_{6}$ derivative and, in addition, the interchain distance $a$ is significantly larger. Consequently, the interchain coupling is weaker. The chemical substitution by larger anions compared to the case of the $\mathrm{PF}_{6}$ derivative can be assimilated to a negative pressure effect and underlines the role of elastic interaction and volume change in the vicinity of phase transition. As a consequence, the transition toward the metallic phase occurs at a lower temper- ature $\left(T_{\mathrm{I}-\mathrm{M}}=242 \mathrm{~K}\right)$ and shows a larger thermal hysteresis $(22 \mathrm{~K})$ for $(\mathrm{EDO}-\mathrm{TTF})_{2} \mathrm{SbF}_{6}$ compared to (EDO-TTF $)_{2} \mathrm{PF}_{6}$ $\left(T_{\mathrm{I}-\mathrm{M}}=278 \mathrm{~K}, 2.5 \mathrm{~K}\right.$ width hysteresis) [10,25]. It can be noted that for all derivatives of the isostructural (EDO-TTF) ${ }_{2} \mathrm{XF}_{6}$ family, the phase transition occurs for almost the same critical value of the D chain cell parameter $b$ and the magnitude of their discontinuous changes at $T_{\mathrm{I}-\mathrm{M}}$ is nearly the same [10]. This is very likely due to the strongly $1 \mathrm{D}$ character of the compound. Since this lattice parameter is larger for the $\mathrm{SbF}_{6}$ derivative, the critical value of $b$ is reached by thermal contraction at lower temperature than for $(\mathrm{EDO}-\mathrm{TTF})_{2} \mathrm{PF}_{6}$. Unlike the intrachain cell parameter, the interchain cell parameters change significantly more as the anion size increases. The anions change their orientation at the phase transition and it requires larger volume change to accommodate movements of bigger anions. Thus, the relative volume jump $\Delta V / V_{\mathrm{HT}}$, where $V_{\mathrm{HT}}$ is the cell volume at high temperature, is three times higher [10] in the case of (EDO-TTF $)_{2} \mathrm{SbF}_{6}$ than in the case of $(\mathrm{EDO}-\mathrm{TTF})_{2} \mathrm{PF}_{6}$. In other words, the first-order character of the phase transition is much stronger for the $\mathrm{SbF}_{6}$ derivative than for the $\mathrm{PF}_{6}$ one. The effect of coupling between the order parameter and the cell deformation is indeed stronger and costs more elastic energy. This is in agreement with the larger thermal hysteresis for (EDO-TTF $)_{2} \mathrm{SbF}_{6}$. The bigger size of anion makes the phase transition more difficult.

The I-M transition and its hysteresis are well observed by magnetic susceptibility $\chi_{m}$ as it vanishes in the I phase by electron pairing [Fig. 2(a)]. It entails an important change of electronic state which leads to significant changes of physical properties and especially of optical reflectivity. The reflectivity spectrum measured with light polarized along the D stacking axis of the crystal in both phases is shown in Fig. 3(a) and indicates that optical reflectivity variations can be used for probing the change of electronic state of this crystal. The relative variation of reflectivity at $1.72 \mathrm{eV}$ with temperature exhibits a clear optical signature of the transition with a hysteretic behavior in perfect agreement with magnetic data

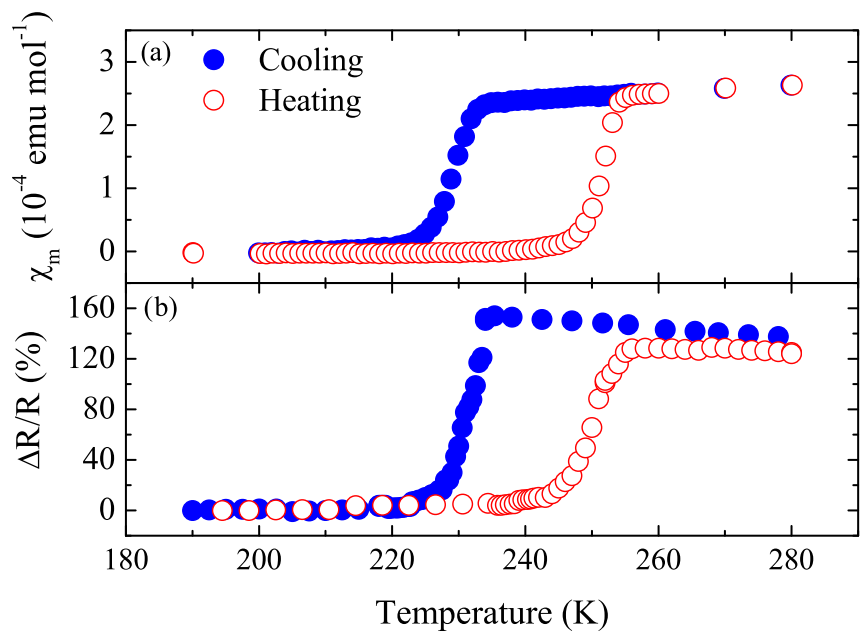

FIG. 2. (Color online) (a) Magnetic susceptibility of $(\mathrm{EDO}-\mathrm{TTF})_{2} \mathrm{SbF}_{6}$ and (b) relative variation of the reflectivity at $1.72 \mathrm{eV}$ as a function of temperature with a polarization parallel to the $\mathrm{D}$ chain (cell axis $b$ ) of $(\mathrm{EDO}-\mathrm{TTF})_{2} \mathrm{SbF}_{6}$ : the hysteresis is centered at 240 and $22 \mathrm{~K}$ wide. 


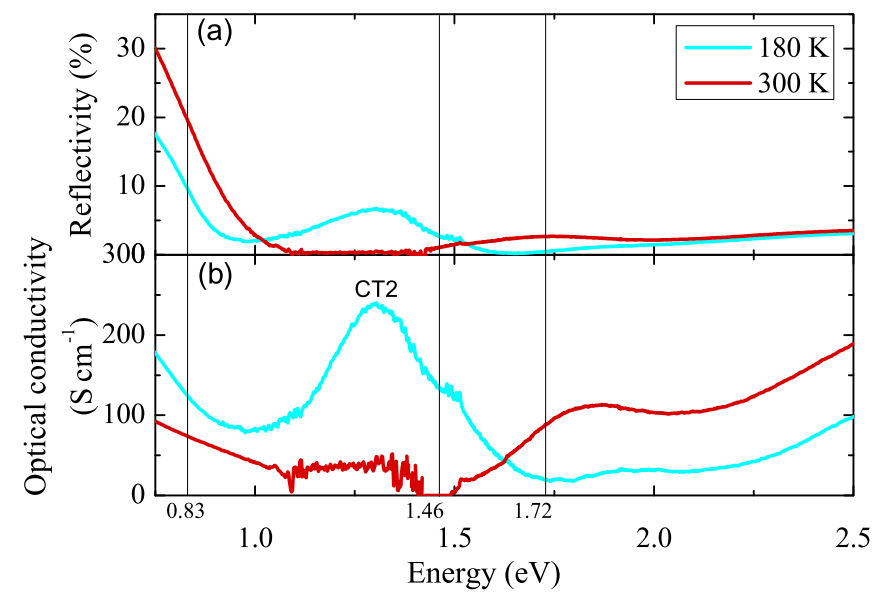

FIG. 3. (Color online) (a) Reflectivity $R$ at different temperatures with a polarisation parallel to the $\mathrm{D}$ stacking axis of $(\mathrm{EDO}-\mathrm{TTF})_{2} \mathrm{SbF}_{6}$. (b) Optical conductivity of (EDO-TTF $)_{2} \mathrm{SbF}_{6}$.

[Fig. 2(b)]. By applying Kramers-Krönig transformation to this reflectivity spectrum, we obtained optical conductivity of the sample [Fig. 3(b)]. The charge transfer (CT) band corresponding to the $\mathrm{CT}$ excitation from $\left(\mathrm{D}^{0} \mathrm{D}^{1} \mathrm{D}^{1} \mathrm{D}^{0}\right)$ to localized $\left\{\mathrm{D}^{0} \mathrm{D}^{2} \mathrm{D}^{0} \mathrm{D}^{0}\right\}$, and referred to as CT2 for (EDO-TTF $)_{2} \mathrm{PF}_{6}[24]$, is observed in the I phase around $1.29 \mathrm{eV}$ [25].

Important changes of Raman spectra are also observed during the phase transition, resulting from the symmetry breaking (splitting of the band) and of the electronic redistribution (changes of frequencies) (Fig. 4). In the $\mathrm{M}$ phase at $250 \mathrm{~K}$, two intense and broad peaks are clearly observed around 1.15 and $2.6 \mathrm{THz}$. In the low temperature I phase, at 100 and $170 \mathrm{~K}$, several modes are easily identified at around 1, 1.5, $2,2.5,2.9,3.2$, and $3.4 \mathrm{THz}$. The increase of the number of Raman active modes comes from the cell doubling, resulting in a doubling of phonon branches, whereas slight softening is observed between 100 and $170 \mathrm{~K}$ in the I phase for most of the modes. In molecular solids, such low frequency modes are attributed to intermolecular vibrations or librations mode, as well as intramolecular modes of boatinglike nature [23,26-30].

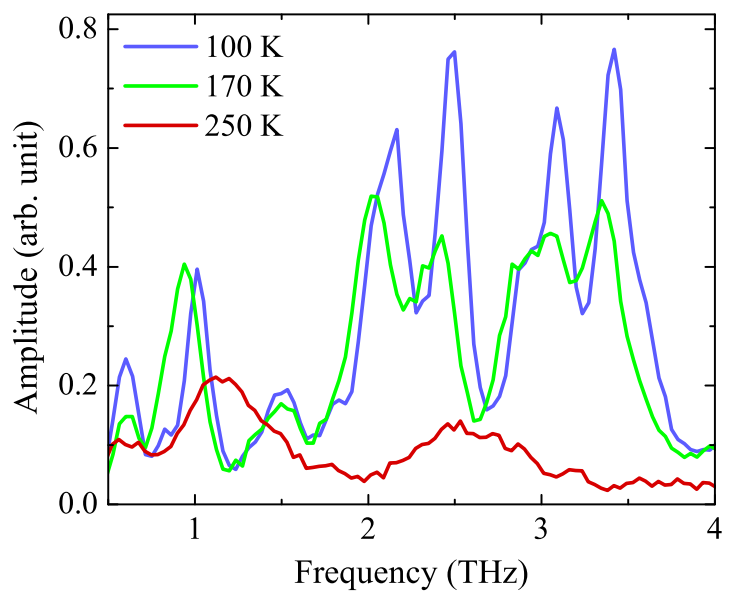

FIG. 4. (Color online) Raman spectra of (EDO-TTF) ${ }_{2} \mathrm{SbF}_{6}$ in the I phase at 100 and $170 \mathrm{~K}$ and in the $\mathrm{M}$ phase at $250 \mathrm{~K}$.

\section{PHOTOINDUCED DYNAMICS}

To gain some insight into the photoinduced dynamics in the family of charge-transfer materials, we investigate here the response to femtosecond laser excitation of (EDO-TTF $)_{2} \mathrm{SbF}_{6}$ in the I phase by using optical pump-probe spectroscopy. The pump energy was $1.46 \mathrm{eV}$, which is nearly resonant with the CT2 band. This scheme of excitation is similar to that used for $(\mathrm{EDO}-\mathrm{TTF})_{2} \mathrm{PF}_{6}$, and it efficiently drives photoinduced transformation. Two probe energies were used: $1.72 \mathrm{eV}$ on the high energy side of the CT2 band, a region with a relatively good reflectivity in the high temperature phase, and $0.83 \mathrm{eV}$ on the high energy side of the CT1 band [25] (Fig. 3).

In order to understand the first steps of the transformation in $(\mathrm{EDO}-\mathrm{TTF})_{2} \mathrm{SbF}_{6}$, the experiments were conducted on the picosecond and nanosecond time scale at different temperatures. Figure 5 shows the typical time traces of the relative reflectivity variation $\Delta R / R$ at pump fluency of $600 \mu \mathrm{J} \mathrm{cm}^{-2}$. It shows an instantaneous peak reflecting the initial electronic photogenerated state $\left\{\mathrm{D}^{0} \mathrm{D}^{2} \mathrm{D}^{0} \mathrm{D}^{0}\right\}$ which decays exponentially in $300 \mathrm{fs}$ (Fig. 6) to a long lived transient state. This state is different from the insulating ground state and persists over at least $3 \mathrm{~ns}$. For the $1.72 \mathrm{eV}$ probe energy, $\Delta R / R$ is positive [Fig. 5(a)], as observed between I and $M$ phase at thermal equilibrium. However, $\Delta R / R$ is negative at $0.83 \mathrm{eV}$ [Fig. 5(b)], whereas it is positive at thermal equilibrium going from I to M phase [Fig. 3(a)]. This is a clear indication that this photoinduced state is different from both the insulating and the high temperature metallic states. This is in agreement with recent femtosecond electron diffraction [9] and infrared [8] studies of (EDO-TTF $)_{2} \mathrm{PF}_{6}$, which revealed the formation of an intermediate state prior to $\mathrm{M}$ phase. This $\left(\mathrm{D}^{0} \mathrm{D}^{1} \mathrm{D}^{0} \mathrm{D}^{1}\right)$ state is characterized also by negative change in the $0.8 \mathrm{eV}$ region of the reflectivity spectrum [8]. Our optical data therefore reveal the photogeneration of the $\left(\mathrm{D}^{0} \mathrm{D}^{1} \mathrm{D}^{0} \mathrm{D}^{1}\right)$ state in $(\mathrm{EDO}-\mathrm{TTF})_{2} \mathrm{SbF}_{6}$.

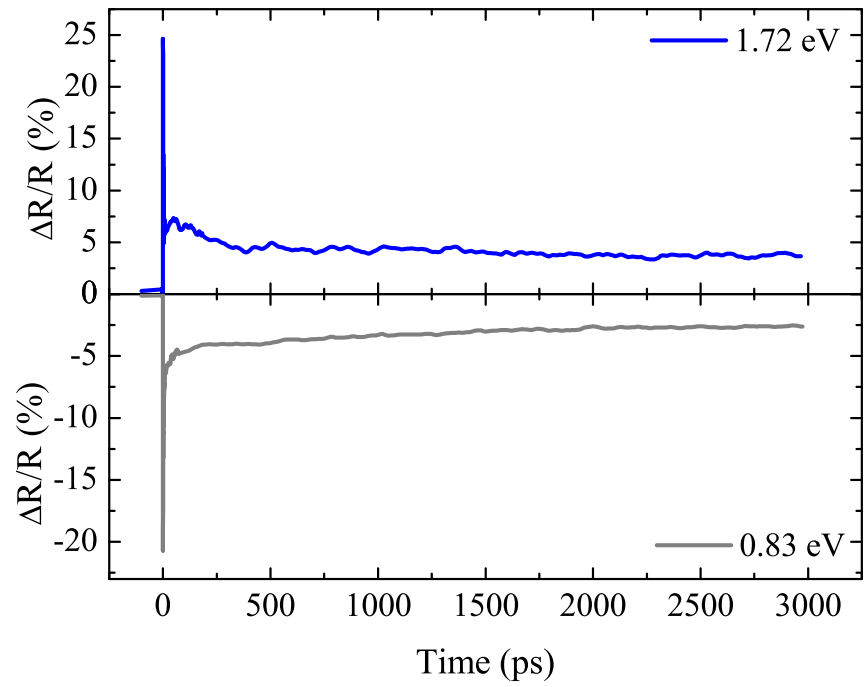

FIG. 5. (Color online) Photoresponse of (EDO-TTF) ${ }_{2} \mathrm{SbF}_{6}$ at $100 \mathrm{~K}$ for a $1.46 \mathrm{eV}$ pump and $1.72 \mathrm{eV}$ probe (upper graph) and $0.83 \mathrm{eV}$ probe (lower graph). 

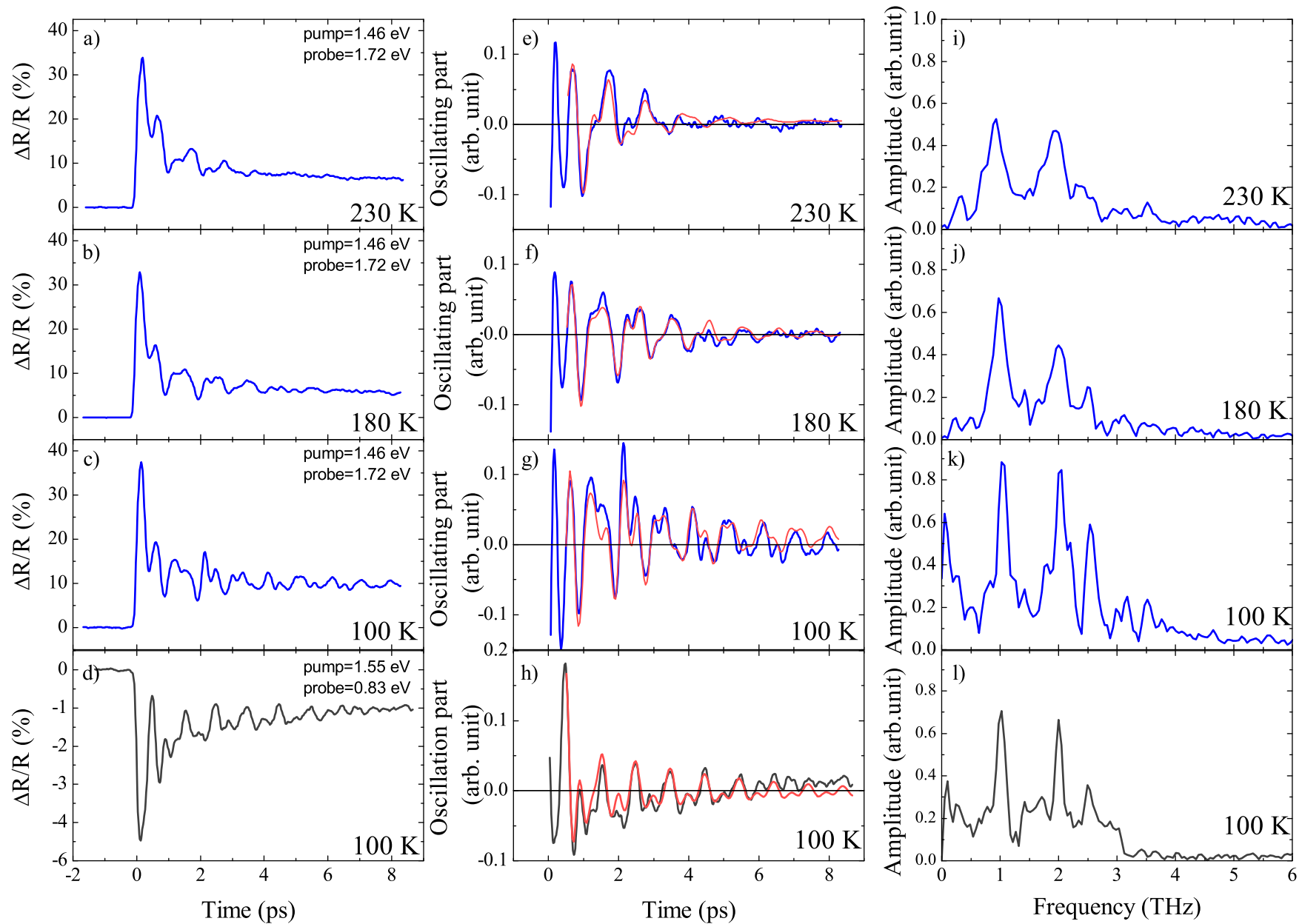

FIG. 6. (Color online) Photoresponse of (EDO-TTF $)_{2} \mathrm{SbF}_{6}$ at 100,180 , and $230 \mathrm{~K}$ for a $1.46 \mathrm{eV}$ pump and $1.72 \mathrm{eV}$ probe (a)-(c) and a $1.55 \mathrm{eV}$ pump and $0.83 \mathrm{eV}$ probe (d). Oscillation part retrieved from the photoresponse and the corresponding fit (thin red or light gray lines) with three phonon modes [(e)-(h) correspond to (a)-(d), respectively]. The transformed Fourier spectra obtained from the oscillation [(i)-(l) correspond to (e)-(h), respectively].

\section{A. Coherent phonons}

The zoom on the time traces of $\Delta R / R$ data in the first $8 \mathrm{ps}$ is shown in Figs. 6(a)-6(d), for which pump fluency was set to $1100 \mu \mathrm{J} \mathrm{cm}^{-2}$. The initial peak which decreases with a $300 \mathrm{fs}$ time constant to the metastable state appears now clearly. A multicomponent oscillation superimposed on the main signal can be observed and unveils some characteristics of the new state. These oscillations result from the simultaneous excitation of several optical coherent phonons as observed in other charge-transfer materials [30,31] and also in spincrossover crystals [32]. We retrieved the oscillating part of the $\Delta R / R$ signal by subtracting the exponential decay of the electronic peak excitation convoluted with the temporal Gaussian for the laser pulse [Figs. 6(e)-6(h)]. By extending the oscillating signal with zero padding on time domain, we performed Hanning windowed Fourier transform with a satisfactory frequency definition [Figs. 6(i)-6(1)]. At $1.72 \mathrm{eV}$, three main modes appear at the different temperatures around 1,2 , and $2.5 \mathrm{THz}$, as well as weaker ones around 1.5, 2.9, 3.2 , and $3.5 \mathrm{THz}$. At $0.83 \mathrm{eV}$, the same main modes are also observed at 1,2 , and $2.5 \mathrm{THz}$. This corroborates the results obtained at $1.72 \mathrm{eV}$.
It can be noted that the global shape of the oscillations looks different depending on the probe energy used: while the mode at $2.9 \mathrm{THz}$ is barely distinguishable, those at 3.2 and $3.5 \mathrm{THz}$ are invisible at $0.83 \mathrm{eV}$ [Fig. 6(1)]. Such ultrafast oscillation of optical reflectivity is generated by the activation of coherent optical phonons, which modulate the dielectric susceptibility through electron-phonon coupling. The contribution of a specific phonon to optical modulation is photon energy dependent [33] and explains the apparent relative change of the spectral weight of the modes for measurements using 0.83 and $1.72 \mathrm{eV}$ probes.

In order to describe more accurately the phonon dynamics, we performed time-dependent analysis by fitting the oscillating component $s(t)$ of the reflectivity signal. The fitting model consists of the sum of three damped cosines corresponding to the three main modes, indexed $i$, of the Fourier spectrum, each characterized by its own amplitude $A_{i}$, frequency $v_{i}$, phase $\varphi_{i}$, and damping rate $\gamma_{i}$ :

$$
s(t)=\sum_{i=1}^{3} A_{i} \cos \left(2 \pi v_{i} t+\varphi_{i}\right) \exp \left(-\gamma_{i} t\right) .
$$



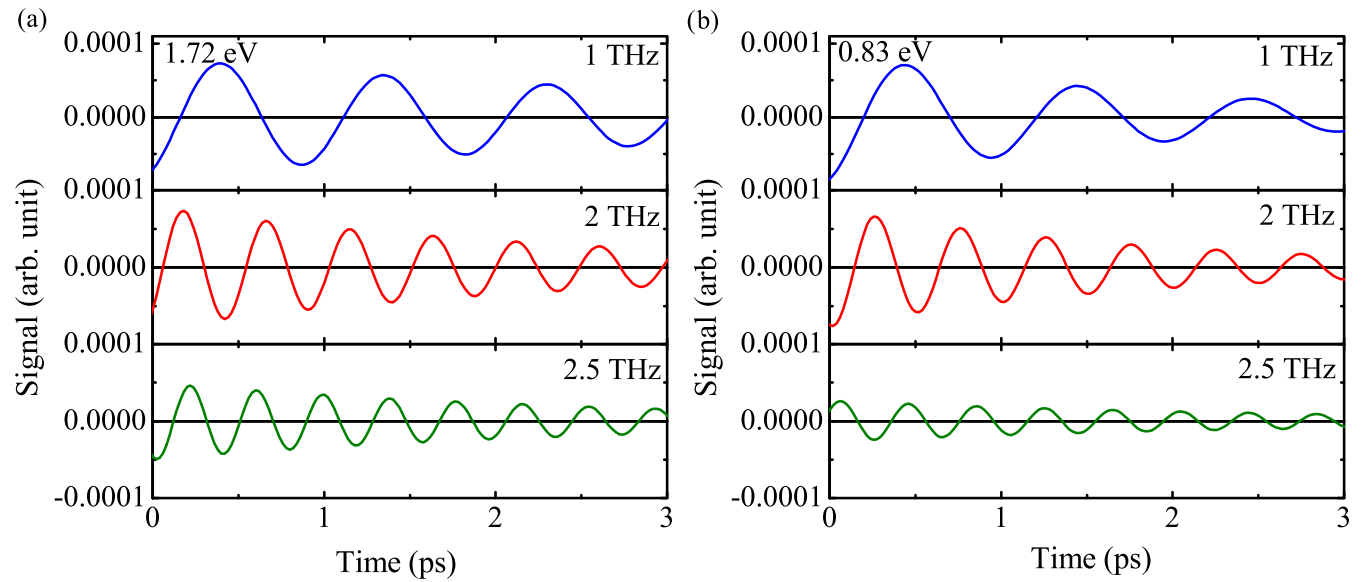

FIG. 7. (Color online) Oscillograms of the three main phonons at $100 \mathrm{~K}$ for a probe at $1.72 \mathrm{eV}$ (a) and $0.83 \mathrm{eV}$ (b).

The oscillations are nicely reproduced [Figs. 6(e)-6(h)] by this model in the temperature range $100-230 \mathrm{~K}$. With this method we extracted the values of the frequencies, damping rates, and initial phases of the three main phonons identified. Figure 7 displays these components independently for the two probe energies at $100 \mathrm{~K}$. For the three modes, the results are almost similar. The contribution of the $2.5 \mathrm{THz}$ phonon to reflectivity change at $0.83 \mathrm{eV}$ appears to be $\pi$ phase shifted compared to that at $1.72 \mathrm{eV}$. The three modes appear to be of cosine type ( $\varphi$ close to 0 ) indicating that immediately after photoexcitation the amplitude is maximum. This is characteristic of a displacive mechanism [34]: the structural reorganization induced by the laser excitation moves the system toward a new minimum position in the potential energy surface for this photoinduced $\left(\mathrm{D}^{0} \mathrm{D}^{1} \mathrm{D}^{0} \mathrm{D}^{1}\right)$ state. This is associated with the displacive nature of the structural trapping, as intra- and intermolecular structure change, directly evidenced by femtosecond electron diffraction in the $\mathrm{PF}_{6}$ derivative [9].

A detailed study of the photoinduced dynamics was then performed by using the $1.46 \mathrm{eV}$ pump and $1.72 \mathrm{eV}$ probe for which the relative increase of reflectivity is larger between $\mathrm{I}$ and $\mathrm{M}$ phases and where the vibrations modes are better observed.

\section{B. Response to different laser fluencies}

First, we investigated the dependence of the photoresponse to excitation densities up to 4 times higher than those used in Ref. [5]. Figure 8(a) shows selected data analyzed as explained above and Fig. 8(b) shows the dependence of the frequencies of the three main modes with the pump fluency. These results indicate that there is very little, if any, dependence of the frequencies with the pump excitation density. This contrasts with the photoexcitation process in systems like bismuth, in which the excited electrons are delocalized over the conduction band and the depopulation of the valence band leads to softening of the interatomic potential, due to thermalization of the electronic and phononic subsystems [35-40]. As a consequence, the $\mathrm{Bi}-\mathrm{Bi}$ dimerization mode softens with the excitation density. This is not the case in our study and this is an indication of the local nature of the structural trapping of the electronic excited state. This local trapping was theoretically introduced to explain the two-step dynamics [6-9], which we will discuss in the last part of this paper.

In addition to the oscillating part discussed above, the photoinduced dynamics is also characterized by an important increase of reflectivity at $1.72 \mathrm{eV}$. Figure 9 shows the excitation density dependence of $\Delta R / R$ integrated on two different time scales: at the picosecond time scale, between 6.5 and $8.5 \mathrm{ps}$ [Fig. 9(a)], and at the nanosecond time scale, between 2.3 and $3 \mathrm{~ns}$ [Fig. 9(b)]. On the picosecond time scale the reflectivity appears to change linearly with excitation density and we could not detect any threshold manifesting nonlinear effects in (EDO-TTF $)_{2} \mathrm{SbF}_{6}$ despite spanning a broad excitation density range. Our measurements were performed with fluencies from 4 to $1400 \mu \mathrm{J} \mathrm{cm}^{-2}$. This range starts from 10
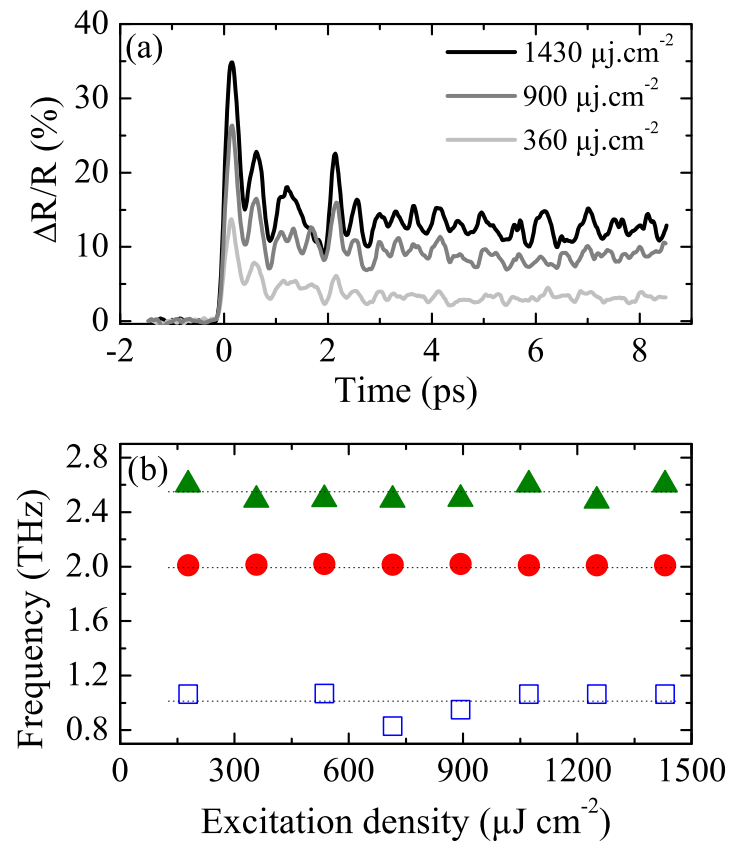

FIG. 8. (Color online) (a) Photoresponse of (EDO-TTF) ${ }_{2} \mathrm{SbF}_{6}$ at $100 \mathrm{~K}$ for $1.46 \mathrm{eV}$ pump and $1.72 \mathrm{eV}$ probe at different fluencies. (b) Photon excitation density dependence of the phonon frequencies at $100 \mathrm{~K}$. 
(a)

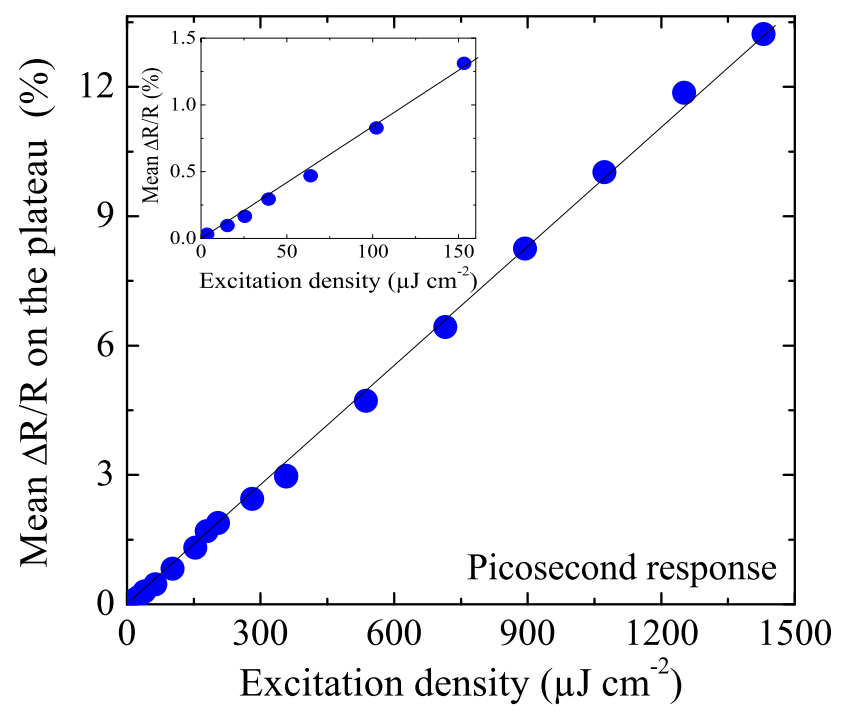

(b)

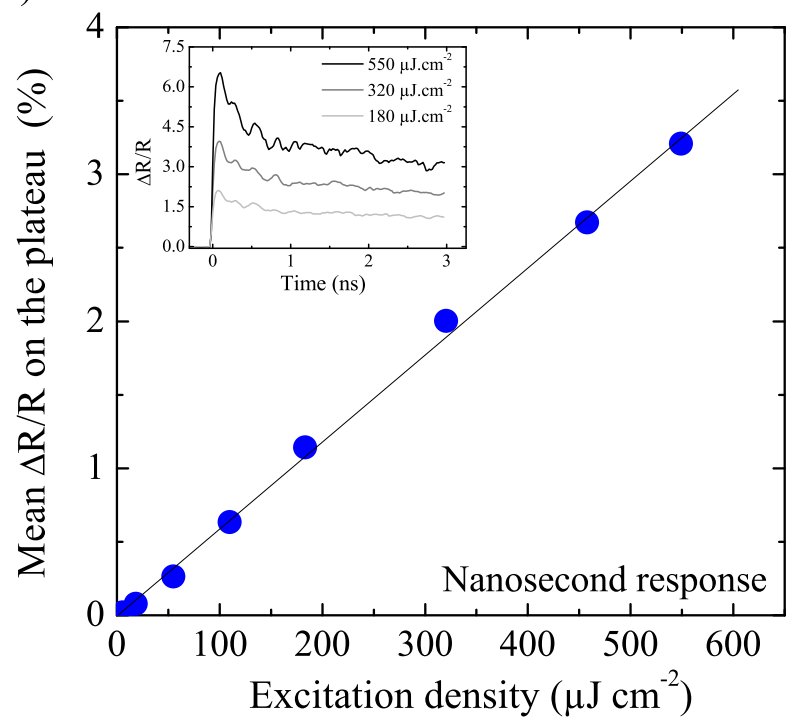

FIG. 9. (Color online) Plateau signal for $1.46 \mathrm{eV}$ pump and $1.72 \mathrm{eV}$ probe, integrated between 6.5 and 8.5 ps at $100 \mathrm{~K}$ (a). The blow up of the excitation densities under $150 \mu \mathrm{J} \mathrm{cm}^{-2}$ is in the inset. Plateau signal integrated between 2.3 and $3 \mathrm{~ns}$ (b). The inset shows the photoresponse at different excitation densities.

times lower than the excitation density threshold determined for (EDO-TTF) ${ }_{2} \mathrm{PF}_{6}$ [5], at around $50 \mu \mathrm{J} \mathrm{cm}^{-2}$, and extends to about 30 times higher, i.e., just before damage. On the nanosecond time scale, $\Delta R / R$ shows oscillations with $100 \mathrm{~s} \mathrm{ps}$ period [inset of Fig. 9(b)]. These are very likely due to acoustic waves and are damped within $1 \mathrm{~ns}$. Therefore, the averaging range from 2.3 to $3 \mathrm{~ns}$ does not take into account these oscillations. Similarly to the picosecond time scale, the photoresponse exhibits a linear response with excitation density [Fig. 9(b)].

\section{Temperature dependence}

Time-resolved optical data were also collected at different temperatures and analyzed the same way. Figure 10(a) shows how frequencies of the main three modes change with temperature. The modes around 1 and $2 \mathrm{THz}$ exhibit a clear
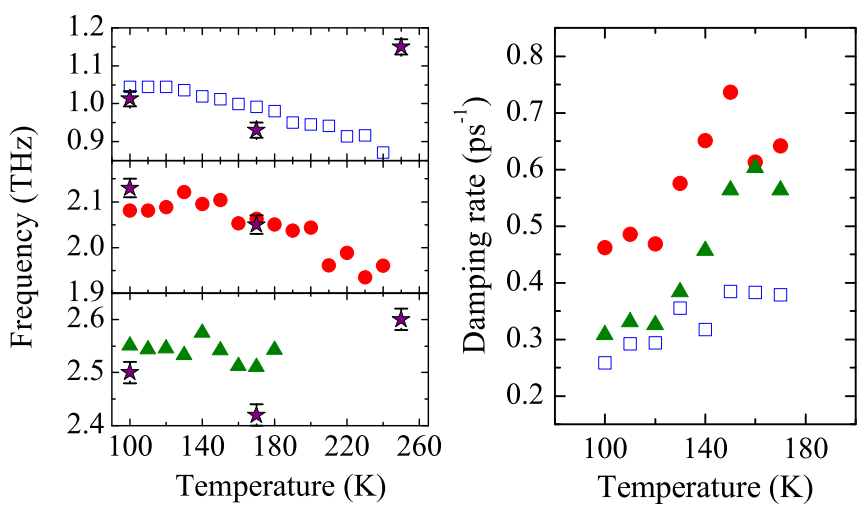

FIG. 10. (Color online) (a) Temperature dependence of frequencies for the three main modes at around $1 \mathrm{THz}(\square), 2 \mathrm{THz}(\bullet)$, and $2.5 \mathrm{THz}(\boldsymbol{\Delta})$ from the fit model and by Raman spectroscopy ( $\star$ ) from 100 to $240 \mathrm{~K}$. (b) Temperature dependence of damping rate. softening with temperature, while that around $2.5 \mathrm{THz}$ is more difficult to analyze because of its weak spectral weight above 190 K. Figure 10(b) shows the damping rate of the modes with temperature below $190 \mathrm{~K}$.

\section{DISCUSSION}

The results presented here for (EDO-TTF $)_{2} \mathrm{SbF}_{6}$ can be compared with the experimental and theoretical reports on the isostructural (EDO-TTF $)_{2} \mathrm{PF}_{6}$ for which a rich literature already exists. The photoexcitation of the initial chargeordered I phase $\left(\mathrm{D}^{0} \mathrm{D}^{1} \mathrm{D}^{1} \mathrm{D}^{0}\right)$ in the $\mathrm{CT}$ band drives a $\mathrm{CT}$ to the $\left\{\mathrm{D}^{0} \mathrm{D}^{2} \mathrm{D}^{0} \mathrm{D}^{0}\right\}$ state which very rapidly relaxes locally to the $\left(\mathrm{D}^{0} \mathrm{D}^{1} \mathrm{D}^{0} \mathrm{D}^{1}\right)$ state. This new local order is trapped by a structural reorganization involving different degrees of freedom. Femtosecond electron diffraction [9] on (EDO-TTF $)_{2} \mathrm{PF}_{6}$ revealed the important role of three main displacements: the bending of EDO-TTF molecules, the sliding of flat EDO-TTF molecules correlated to the sliding of the anion. The displacive nature of this local trapping drives the system towards a local minimum of the potential energy surface and these three different coordinates oscillate in a new potential. Since we observe three main modes we tentatively assign them to the motions observed by electron diffraction but it is difficult to attribute the vibrations observed to specific motions.

On the picosecond time scale, the amplitude of the photoresponse changes linearly with excitation density of the pump laser [Fig. 9(a)], whereas the mode frequencies remain unchanged [Fig. 8(b)]. These two features agree with a local nature of the initial process similarly with the $\mathrm{PF}_{6}$ derivative [6]. In parallel, the frequencies of the observed modes reported in Fig. 10(a) differ from those measured at thermal equilibrium in the I phase. This indicates that the process observed does not correspond to an impulsive Raman process. These modes are different too from those of the $\mathrm{M}$ phase and this is spectroscopic evidence that the 
M phase is not formed on a picosecond time scale. This is confirmed by the fact that $\Delta R / R$ remains negative at $0.83 \mathrm{eV}$ [Fig. 6(d)], whereas it is positive in the thermal M phase [Fig. 3(a)]. We conclude that this state is similar to the transient intermediate state formed on the picosecond time scale in (EDO-TTF) ${ }_{2} \mathrm{PF}_{6}$ and tentatively assigned it to the local $\left(\mathrm{D}^{0} \mathrm{D}^{1} \mathrm{D}^{0} \mathrm{D}^{1}\right)$ state [6]. Finally, this initial step, taking place at the molecular level, does not seem affected by bigger anion size as the photoresponse is similar for both compounds.

On the 100-ps time scale, (EDO-TTF $)_{2} \mathrm{PF}_{6}$ exhibits a clear change of structure and charge revealed by the structural dynamics studied both by ultrafast vibrational spectroscopy [8] and ultrafast electrons diffraction [9]. These changes unveil the formation of a state similar to the thermal $\mathrm{M}\left(\mathrm{D}^{0.5} \mathrm{D}^{0.5} \mathrm{D}^{0.5} \mathrm{D}^{0.5}\right)$ phase. They are accompanied by modification of the infrared spectroscopic fingerprint, approaching that of the $\mathrm{M}$ phase [8]. This is not the case in the present (EDO-TTF $)_{2} \mathrm{SbF}_{6}$ compound, for which the $\Delta R / R$ at $0.83 \mathrm{eV}$ remains clearly negative and of the same order of magnitude until $3 \mathrm{~ns}$ (Fig. 5), whereas it should be positive if the M phase was formed [Fig. 3(a)]. We could not detect any formation of the metallic state on the nanosecond time scale, but only a decay of this photoinduced state as shown in Fig. 5, even for excitation densities close to sample damage and higher than that used for $(\mathrm{EDO}-\mathrm{TTF})_{2} \mathrm{PF}_{6}$. We conclude that unlike (EDO-TTF $)_{2} \mathrm{PF}_{6},(\mathrm{EDO}-\mathrm{TTF})_{2} \mathrm{SbF}_{6}$ does not undergo conversion towards the $\mathrm{M}$ phase and shows exclusively local response to light excitation. With such local response, a saturation regime should be observed when the number of photons equals the number of molecules. However, the sample degradation occurs at $1500 \mu \mathrm{J} \mathrm{cm}^{-2}$ (1 photon per 50 molecules). Therefore, we are far from the saturation regime and this is consistent with the observed linear response. As the only structural difference between the two compounds is the larger size of $\mathrm{SbF}_{6}$ anions resulting in a three times larger $\Delta V / V_{\mathrm{HT}}$ during the I-M transition, we conclude that the phase transition towards the metallic phase is more difficult because it costs more elastic energy for the $\mathrm{SbF}_{6}$ than the $\mathrm{PF}_{6}$ derivative. It is established now that during the early stage of the photoinduced process, the volume does not change. The volume expansion, which is required for driving macroscopic phase change, typically occurs on an acoustic time scale defined by the ratio between the laser penetration depth and the speed of sound [21]. We note that the establishment of the M phase in the $\mathrm{PF}_{6}$ derivative occurs only around $100 \mathrm{ps}[8,9]$. Despite the occurrence of thermal phase transition in (EDO-TTF $)_{2} \mathrm{SbF}_{6}$, the associated volume change is too large to be overcome during the photoinduced process and this prevents the formation of a macroscopic three-dimensional $\mathrm{M}$ order. This picture is consistent with our study which indicates that the photoinduced transformation remains local in this compound.

\section{SUMMARY}

The counteranion in the family of (EDO-TTF $)_{2} \mathrm{XF}_{6}$ compounds has always been considered to be of prime importance in the thermal transition [11] and its role in the photoinduced transformation was evoked by recent time-resolved electron diffraction experiments. However, until now, the fundamental importance of its size in photoinduced phenomena was not discussed. In this work we investigate the photoinduced transformation of the (EDO-TTF $)_{2} \mathrm{SbF}_{6}$ derivative with a bigger counteranion. It acts as a negative pressure which increases the distance between chains and decreases the coupling between them. We observe that the photoinduced state is unambiguously of local nature as it is the case for the initial state. The major new finding is that $(\mathrm{EDO}-\mathrm{TTF})_{2} \mathrm{SbF}_{6}$ does not reach the photoinduced $\mathrm{M}$ phase, which we rationalize with hindrance by the larger counteranions.

\section{ACKNOWLEDGMENTS}

This work was supported by the Institut Universitaire de France, the ANR (ANR-13-BS04-0002), Région Bretagne (CREATE 4146), Rennes Métropole, Fonds Européen de Développement Régional (FEDER), and JSPS KAKENHI Grant No. 15K17901 and 26288035. Y.N. received a research grant from the JGC-S Scholarship Foundation.
[1] H. Cailleau, M. Lorenc, M. Buron-Le Cointe, M. Servol, M. Cammarata, and E. Collet, Eur. Phys. J. Special Topics 222, 1077 (2013).

[2] H. Cailleau, T. Luty, S. Koshihara, M. Servol, M. Lorenc, M. Buron-Le Cointe, and E. Collet, Acta Phys. Pol. A 121, 297 (2012).

[3] K. Yonemitsu and K. Nasu, Phys. Rep. 465, 1 (2008).

[4] K. Nasu, Photoinduced Phase Transitions, edited by K. Nasu (World Scientific, Singapore, 2004).

[5] M. Chollet, L. Guerin, N. Uchida, S. Fukaya, H. Shimoda, T. Ishikawa, K. Matsuda, T. Hasegawa, A. Ota, H. Yamochi, G. Saito, R. Tazaki, S. Adachi, and S. Koshihara, Science 307, 86 (2005).

[6] K. Onda, S. Ogihara, K. Yonemitsu, N. Maeshima, T. Ishikawa, Y. Okimoto, X. Shao, Y. Nakano, H. Yamochi, G. Saito, and S. Koshihara, Phys. Rev. Lett. 101, 067403 (2008).

[7] Y. Matsubara, S. Ogihara, J. Itatani, N. Maeshima, K. Yonemitsu, T. Ishikawa, Y. Okimoto, S. Koshihara, T. Hiramatsu, Y. Nakano,
H. Yamochi, G. Saito, and K. Onda, Phys. Rev. B 89, 161102 (2014).

[8] N. Fukazawa, M. Shimizu, T. Ishikawa, Y. Okimoto, S. Koshihara, T. Hiramatsu, Y. Nakano, H. Yamochi, G. Saito, and K. Onda, J. Phys. Chem. C 116, 5892 (2012).

[9] M. Gao, C. Lu, H. Jean-Ruel, L. C. Liu, A. Marx, K. Onda, S. Koshihara, Y. Nakano, X. Shao, T. Hiramatsu, G. Saito, H. Yamochi, R. R. Cooney, G. Moriena, G. Sciaini, and R. J. D. Miller, Nature (London) 496, 343 (2013).

[10] Y. Nakano, H. Yamochi, G. Saito, M. Uruichi, and K. Yakushi, J. Phys.: Conf. Ser. 148, 012007 (2009).

[11] A. Ota, H. Yamochi, and G. Saito, J. Mater. Chem. 12, 2600 (2002).

[12] S. Aoyagi, K. Kato, A. Ota, H. Yamochi, G. Saito, H. Suematsu, M. Sakata, and M. Takata, Angew. Chem. 116, 3756 (2004).

[13] K. Iwano and Y. Shimoi, Phys. Rev. B 77, 075120 (2008).

[14] K. Onda, H. Yamochi, and S. Koshihara, Acc. Chem. Res. 47, 3494 (2014). 
[15] K. Iwano and Y. Shimoi, Phys. Rev. Lett. 110, 116401 (2013).

[16] K. Yonemitsu and N. Maeshima, Phys. Rev. B 76, 075105 (2007).

[17] G.-J. Linker, P. H. M. van Loosdrecht, P. van Duijnen, and R. Broer, Chem. Phys. Lett. 487, 220 (2010).

[18] CrysAlis RED, Oxford Diffraction Ltd, Version 1.171.32.5 (2007).

[19] A. Altomare, M. C. Burla, M. Camalli, G. Cascareno, C. Giacovazzo, A. Guagliardi, A. G. G. Moliterni, G. Polidori, and R. Spagna, J. Appl. Crystallogr. 32, 115 (1999).

[20] G. M. Sheldrick, Acta Crystallogr. Sect. A 64, 112 (2008).

[21] M. Lorenc, C. Balde, W. Kaszub, A. Tissot, N. Moisan, M. Servol, M. Buron-LeCointe, H. Cailleau, P. Chasle, P. Czarnecki, M. L. Boillot, and E. Collet, Phys. Rev. B 85, 054302 (2012).

[22] CCDC-1023744 (250 K) and CCDC-1023745 (150 K) contain the supplementary crystallographic data for this paper. These data can be obtained free of charge via www.ccdc.cam.ac.uk/ conts/retrieving.html(or from the Cambridge Crystallographic Data Centre, 12 Union Road, Cambridge CB21EZ, UK; fax: (+44) 1223-336033; or deposit@ccdc.cam.uk).

[23] O. Drozdova, K. Yakushi, K. Yamamoto, A. Ota, H. Yamochi, G. Saito, H. Tashiro, and D. B. Tanner, Phys. Rev. B 70, 075107 (2004).

[24] O. Drozdova, K. Yakushi, A. Ota, H. Yamochi, and G. Saito, Synth. Met. 133-134, 277 (2003).

[25] M. Maesato, Y. Nakano, X. Shao, Y. Yoshida, H. Yamochi, G. Saito, A. Moreac, J.-C. Ameline, E. Collet, M. Uruichi, and K. Yakushi, J. Phys.: Conf. Ser. 148, 012004 (2009).

[26] H. Hahn, in Proceedings of the Symposium on Inelastic Scattering of Neutrons, International Atomic Energy Agency, 1965, p. 279.

[27] D. Bormann, J. L. Sauvajol, C. Goze, F. Rachdi, A. Moreac, A. Girard, L. Forro, and O. Chauvet, Phys. Rev. B 54, 14139 (1996).

[28] P. Ranzieri, M. Masino, A. Girlando, and M.-H. Lemée-Cailleau, Phys. Rev. B 76, 134115 (2007).

[29] C. Katan, P. E. Blöchl, P. Margl, and C. Koenig, Phys. Rev. B 53, 12112 (1996).
[30] H. Uemura and H. Okamoto, Phys. Rev. Lett. 105, 258302 (2010).

[31] Y. Kawakami, S. Iwai, T. Fukatsu, M. Miura, N. Yoneyama, T. Sasaki, and N. Kobayashi, Phys. Rev. Lett. 103, 066403 (2009).

[32] M. Cammarata, R. Bertoni, M. Lorenc, H. Cailleau, S. Di Matteo, C. Mauriac, S. F. Matar, H. Lemke, M. Chollet, S. Ravy, C. Laulhé, J.-F. Létard, and E. Collet, Phys. Rev. Lett. 113, 227402 (2014).

[33] K. Onda, S. Ogihara, T. Ishikawa, Y. Okimoto, X. Shao, H. Yamochi, G. Saito, and S. Koshihara, J. Phys. Condens. Matter 20, 224018 (2008).

[34] H. J. Zeiger, J. Vidal, T. K. Cheng, E. P. Ippen, G. Dresselhaus, and M. S. Dresselhaus, Phys. Rev. B 45, 768 (1992).

[35] M. F. DeCamp, D. A. Reis, P. H. Bucksbaum, and R. Merlin, Phys. Rev. B 64, 092301 (2001).

[36] M. Hase, M. Kitajima, S. Nakashima, and K. Mizoguchi, Phys. Rev. Lett. 88, 067401 (2002).

[37] É. D. Murray, D. M. Fritz, J. K. Wahlstrand, S. Fahy, and D. A. Reis, Phys. Rev. B 72, 060301 (2005).

[38] D. M. Fritz, D. A. Reis, B. Adams, R. A. Akre, J. Arthur, C. Blome, P. H. Bucksbaum, A. L. Cavalieri, S. Engemann, S. Fahy, R. W. Falcone, P. H. Fuoss, K. J. Gaffney, M. J. George, J. Hajdu, M. P. Hertlein, P. B. Hillyard, M. Horn-Von Hoegen, M. Kammler, J. Kaspar, R. Kienberger, P. Krejcik, S. H. Lee, A. M. Lindenberg, B. McFarland, D. Meyer, T. Montagne, É. D. Murray, A. J. Nelson, M. Nicoul, R. Pahl, J. Rudati, H. Schlarb, D. P. Siddons, K. Sokolowski-Tinten, T. Tschentscher, D. Von Der Linde, and J. B. Hastings, Science 315, 633 (2007).

[39] K. Sokolowski-Tinten, C. Blome, J. Blums, A. Cavalleri, C. Dietrich, A. Tarasevitch, I. Uschmann, E. Förster, M. Kammler, M. Horn-von-Hoegen, and D. von der Linde, Nature (London) 422, 287 (2003).

[40] W. Lu, M. Nicoul, U. Shymanovitch, A. Tarasevitch, M. Kammler, M. Horn von Hoegen, D. von der Linde, K. Sokolowski-Tinten, in Ultrafast Phenomena XVII, edited by M. Chergui, D. Jonas, E. Riedle, R. Schoenlein, and A. Taylor (Oxford University Press, Oxford, 2011), p. 314. 